\title{
EVALUATION OF CONGENITAL BROWN'S SYNDROME WITH MAGNETIC RESONANCE IMAGING
}

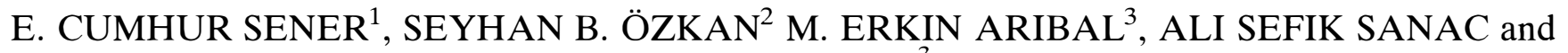 \\ BELMA ASLAN ${ }^{3}$ \\ Ankara, Turkey
}

\begin{abstract}
SUMMARY
The magnetic resonance (MR) findings in congenital Brown's syndrome were investigated. Sixteen involved eyes of 12 patients were compared with 20 eyes of 10 controls. An enlargement of the tendon-trochlea complex was observed in congenital Brown's syndrome. In addition, the appearance of the tendon-trochlea complex was irregular in shape and of intermediate signal intensity. The MR appearances in patients with a satisfactory or poor response to surgery did not show any significant differences from each other or from those in patients who had not undergone surgery. This was also the case for the patient with spontaneous improvement. The MR examination in congenital Brown's syndrome suggested that the abnormality was located at the tendon-trochlea complex but clinically it does not give a clue about the outcome of the disease.
\end{abstract}

Brown's syndrome is an ocular motility disorder characterised by deficient elevation in adduction. It may be congenital or acquired following trauma surgery or arthritis. ${ }^{1-7}$ In the congenital form of the disease, originally described as 'true Brown's syndrome', the short sheath of the superior oblique (SO) tendon was thought to be the aetiological factor, but later detailed anatomical studies showed that such a sheath does not exist. ${ }^{2,8,9}$ The shortness of the SO tendon and/or pathology in the tendon-trochlea complex was thought to be responsible for the clinical picture.

Tenotomy, tenectomy, lengthening and recession of SO tendon are the methods of choice for surgical treatment of Brown's syndrome. However, the

From: ${ }^{1}$ Department of Ophthalmology, Hacettepe University Medical School, Ankara, Turkey; ${ }^{2}$ Department of Ophthalmology, S.B. Ankara Hospital, Ankara, Turkey; ${ }^{3}$ Emaray Medical Imaging Center, Ankara, Turkey.

Correspondence to: Seyhan B. Özkan, MD, Tunali Hilmi cad. 93/11, Kavaklidere, 06700 Ankara, Turkey. variability of response to surgery remains a problem. Hypo- or hypercorrection may frequently occur and it is known that some cases may improve spontaneously. ${ }^{10-12}$ An audible or palpable click was stated to be a preceding sign of spontaneous improvement; however, it was reported that limitation of elevation in adduction may disappear in the absence of the click sign. ${ }^{10,12}$ There is no well-defined sign that gives any clue about the response to surgery.

The aim of this study was to visualise the tendontrochlea complex with magnetic resonance (MR) imaging in congenital Brown's syndrome and to find out whether it gives any predictive clue about the surgical outcome or spontaneous course of the disease.

\section{MATERIALS AND METHODS}

Twelve patients with a clinical diagnosis of congenital Brown's syndrome were included in the study. The diagnostic criteria were as follows: deficient elevation in adduction, less elevation deficiency in midline, minimal or no elevation deficiency in abduction, minimal or no SO overaction and (if it could be performed) a positive traction test. Patients who had had previous SO tenotomy or showed spontaneous improvement were also included.

The study was initiated following approval from the university ethics committee. A detailed description of the study was given to the patients or their parents and a consent form was signed prior to the MR examination. In young patients the MR examination was performed during their sleeping phase and no medication was administered before or during the study.

Orbital MR examinations were performed on a 1.0 tesla superconducting magnet (Picker International, Highland Heights, $\mathrm{OH}$ ) with a surface coil that

Eye (1996) 10, 492-496 co 1996 Royal College of Ophthalmologists 
Table I. The MR measurements of tendon-trochlea complex in patients wiht Brown's syndrome

\begin{tabular}{|c|c|c|c|c|c|c|c|c|c|c|c|}
\hline \multirow{3}{*}{$\begin{array}{c}\text { Patient } \\
\text { no. }\end{array}$} & \multirow{3}{*}{ Involvement } & \multicolumn{8}{|c|}{ Tendon-trochlea complex $(\mathrm{mm})$} & \multirow{3}{*}{$\begin{array}{l}\text { Previous } \\
\text { operation }\end{array}$} & \multirow[b]{3}{*}{ Response to surgery } \\
\hline & & \multicolumn{4}{|c|}{$\mathrm{R}$} & \multicolumn{4}{|c|}{$\mathrm{L}$} & & \\
\hline & & $\mathrm{CC}$ & $\mathrm{W}$ & A & SI & $\mathrm{CC}$ & $\mathrm{W}$ & A & SI & & \\
\hline 1 & $\mathrm{R}$ & 2.9 & 5.2 & I & Hypo & 3 & 3.6 & $\mathrm{Rg}$ & Hypo & - & \\
\hline 2 & $\mathrm{Bi}$ & 2.9 & 4.1 & I & Iso & 2.9 & 3.8 & I & Iso & - & \\
\hline 3 & $\mathrm{~L}$ & 3.1 & 3.7 & $\mathrm{Rg}$ & Hypo & 4.7 & 5.7 & $\mathrm{Rg}$ & Нypo & - & \\
\hline 4 & $\mathrm{Bi}$ & 3.9 & 5.1 & I & Iso & 4.1 & 4.9 & I & Iso & Bil SO & $\mathrm{R}$ overcorrection, $\mathrm{L}$ excellent \\
\hline 5 & $\mathrm{R}$ & 3.5 & 3.7 & I & Iso & 3.5 & 3.4 & $\operatorname{Rg}$ & Нуро & - & \\
\hline 6 & $\mathrm{Bi}$ & 3.3 & 4 & I & Iso & 3.3 & 3.4 & I & Iso & - & \\
\hline 7 & $\mathrm{Bi}$ & 3 & 3.4 & I & Iso & 3.9 & 4.1 & I & Iso & R SO & Good \\
\hline 8 & $\mathrm{R}$ & 2.8 & 3.8 & I & Hypo & 3 & 3.1 & $\mathrm{Rg}$ & Нypo & R SO & Overcorrection \\
\hline 9 & $\mathrm{R}$ & 3.9 & 4.6 & $\mathrm{Rg}$ & Нуро & 3.8 & 4.2 & $\mathrm{Rg}$ & Нуро & R SO & No response \\
\hline 10 & $\mathrm{R}$ & 3.9 & 4.8 & I & Iso & 3.3 & 3.2 & $\mathrm{Rg}$ & Iso & - & Spontaneous improvement \\
\hline 11 & $\mathrm{~L}$ & 3.5 & 4.5 & $\operatorname{Rg}$ & Нуро & 3.7 & 4.5 & I & Iso & - & \\
\hline 12 & $\mathrm{R}$ & 2.9 & 3 & I & Нуро & 2.9 & 2.8 & $\mathrm{Rg}$ & Нуро & - & \\
\hline
\end{tabular}

CC, craniocaudal; W, width, A, appearance; SI, signal intensity; I, irregular; Rg, regular; Hypo, hypointense; Iso, isointense, Bi, bilateral; $\mathrm{SO}$, superior oblique tenotomy.

covered both eyes. In all patients spin echo (SE) T1weighted (640/22 ms TR/TE) contiguous $2 \mathrm{~mm}$ thick multislice images in the axial oblique, sagittal oblique and coronal planes were obtained. Imaging variables included a $200 \times 256$ acquisition matrix and an $18 \mathrm{~cm}$ field of view.

Axial oblique slices were positioned parallel to the SO muscle course. To obtain the most accurate positioning, sagittal pilot images were taken. The best sagittal pilot image for viewing the muscle was chosen as the reference plane. The coronal images were taken perpendicular to an imaginary axis that passed through the orbital apex and the centre of the globe on sagittal pilot views. To provide the basis for this protocol two volunteers were examined with MR prior to the study.

All measurements were done on the monitor independently by two radiologists who were not aware of the site of involvement. The tendontrochlea complex was measured on axial oblique and coronal slices. Ten patients who underwent MR examination for unrelated reasons were used as controls. The ages of the controls and the patients were compared by Mann-Whitney $U$-test.

Student's $t$-test was used for statistical analysis of the measurements and any $p$ value less than 0.05 was accepted as significant.

\section{RESULTS}

Twelve patients ( 6 male, 6 female) who were clinically proven to have Brown's syndrome were examined. The median of the age of the patients was 7 years (range 3-18 years). The controls (5 male, 5 female) had a median age of 7.5 years (range 3-19 years). The ages of the control and study groups were similar $(p=0.000)$.

Four patients were found to have bilateral Brown's syndrome and a total of 16 affected eyes were examined (Table I; Fig. 1). The mean of the craniocaudal measurements was $3.48 \pm 0.56 \mathrm{~mm}$ in affected eyes and $2.76 \pm 0.26 \mathrm{~mm}$ in controls (Table
II). The difference between these groups was significant $(p=0.000)$.

The mean width of the tendon-trochlea complex was $4.26 \pm 0.75 \mathrm{~mm}$ in affected eyes and $3.07 \pm 0.36 \mathrm{~mm}$ in controls $(p=0.000)$ (Figs. 2-4). The appearance and signal intensity of tendontrochlea complex were evaluated and compared with controls. In 14 of the affected eyes the appearance of the tendon-trochlea complex was irregular. In 11 of the affected eyes the tendontrochlea complex was isointense to soft tissue and in all these cases was irregular in shape. The control group had a tendon-trochlea complex of regular appearance and low signal intensity.

Patient 10 showed spontaneous improvement. He was found to have a -1 elevation deficiency in adduction at his last examination, whereas his earlier medical records had shown an elevation deficiency of -4 . He underwent MR examination during his last visit and was found to have an enlarged tendontrochlea complex in both coronal and axial planes (Fig. 3). The complex was irregular in shape and of intermediate signal intensity on the involved side.

In patient 9 , in whom there was no response to surgery, the tendon-trochlea complex was enlarged but appeared regular and hypointense. In the right

Table II. MR measurements of tendon-trochlea complex in control group

\begin{tabular}{llllll}
\hline & \multicolumn{3}{c}{ Tendon-trochlea complex $(\mathrm{mm})$} \\
\cline { 2 - 3 } \cline { 5 - 6 } \cline { 5 - 6 } Patient no. & $\mathrm{CC}$ & $\mathrm{K}$ & & $\mathrm{C}$ & $\mathrm{L}$ \\
\hline 1 & 2.8 & 3.8 & & 2.7 & $\mathrm{~W}$ \\
\hline & 2.7 & 2.3 & & 3 & 3.5 \\
3 & 2.6 & 3.2 & & 2.8 & 3.5 \\
4 & 2.3 & 3.1 & & 2.1 & 3.3 \\
5 & 2.9 & 3.4 & & 3.2 & 3.5 \\
6 & 2.6 & 2.7 & & 2.7 & 2.8 \\
7 & 3 & 3 & & 3 & 3 \\
8 & 3 & 3 & & 2.9 & 3.1 \\
9 & 2.8 & 3 & & 2.9 & 2.9 \\
10 & 2.6 & 3 & & 2.5 & 2.8 \\
\hline
\end{tabular}

CC, craniocaudal; $\mathrm{W}$, width. 


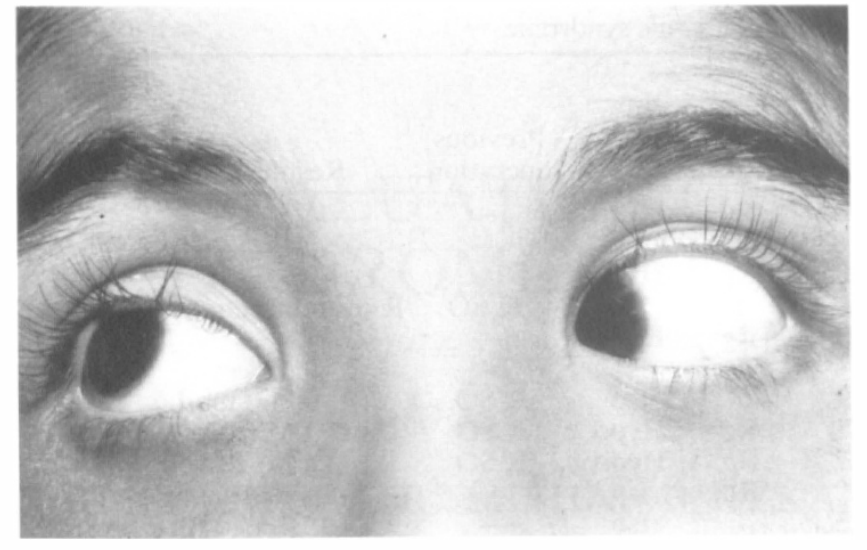

(a)

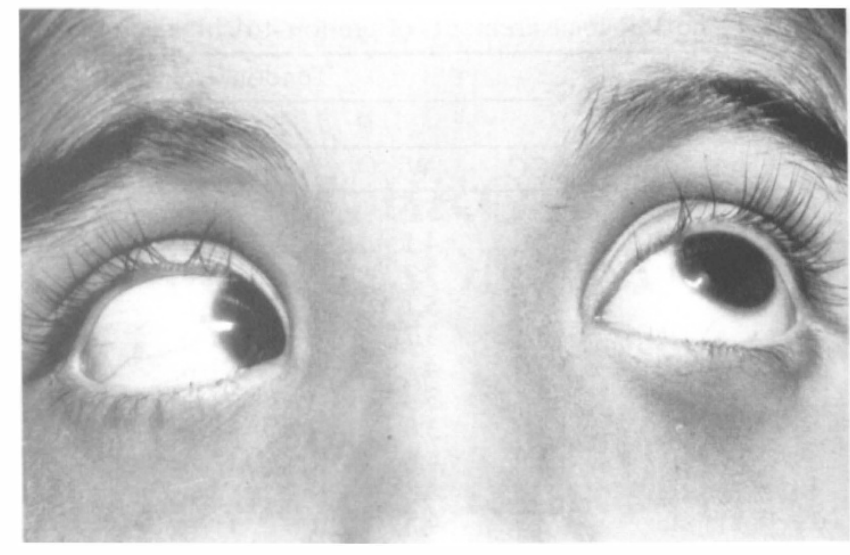

(b)

Fig. 1. Patient 4. Bilateral congenital Brown's syndrome. (a) Deficient elevation of the left eye in adduction, (b) deficient elevation of the right eye in adduction.

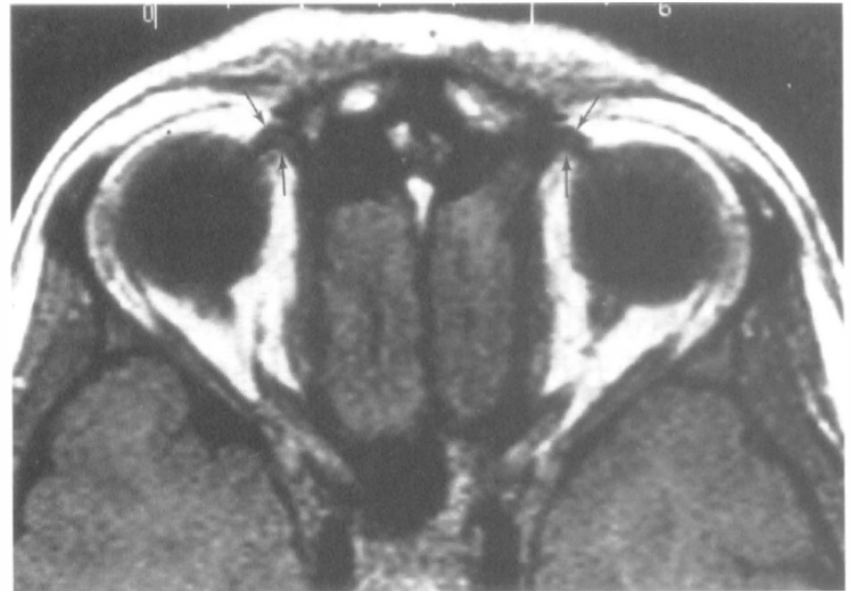

(a)

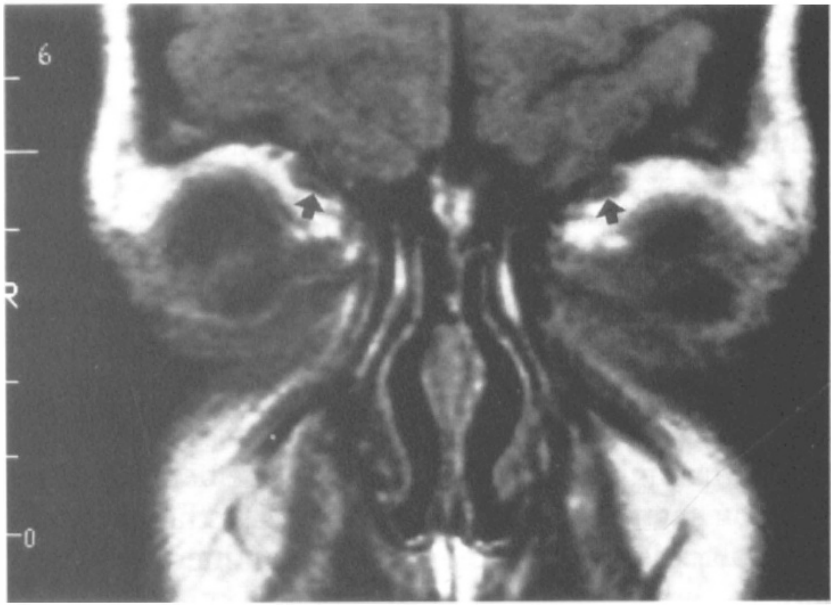

(b)

Fig. 2. Normal appearance of tendon-trochlea complex (arrows) in a control patient. The axial oblique (a) and coronal (b) images display the regular and hypointense tendon-trochlea complex of both orbits.

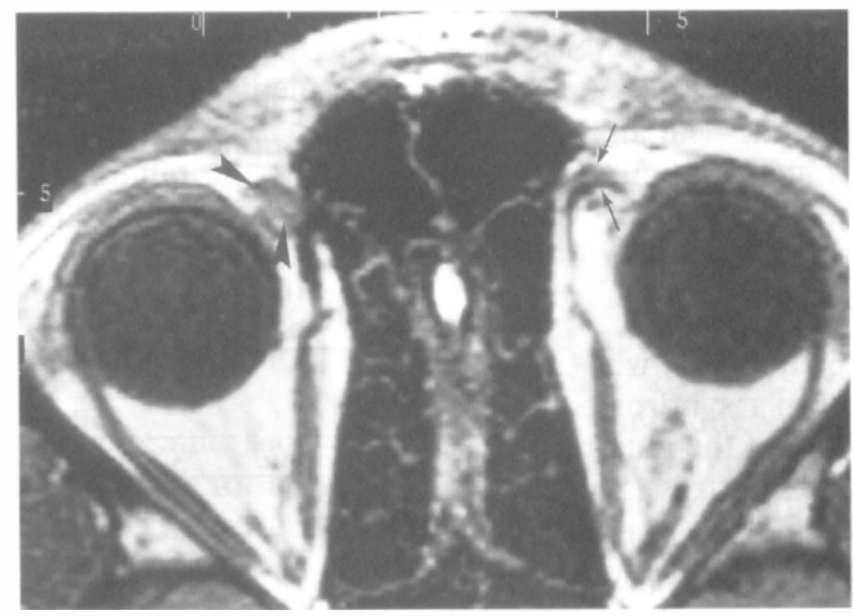

Fig. 3. Patient 10. The axial oblique image in right Brown's syndrome. The tendon-trochlea complex (arrowheads) of the right orbit is irregular and isointense with soft tissue. Note the regularity and low signal intensity of the tendon-trochlea complex (arrows) of the normal left orbit. 


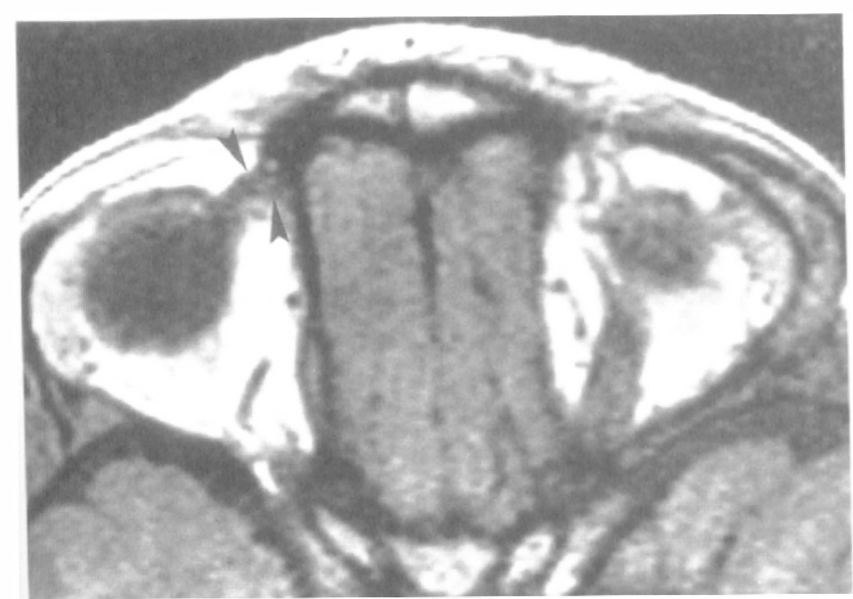

(a)

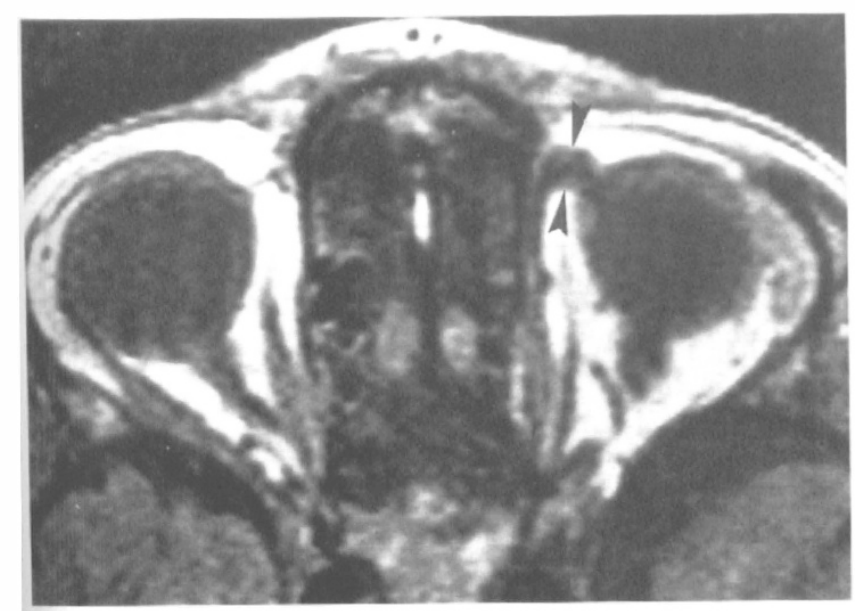

(c)

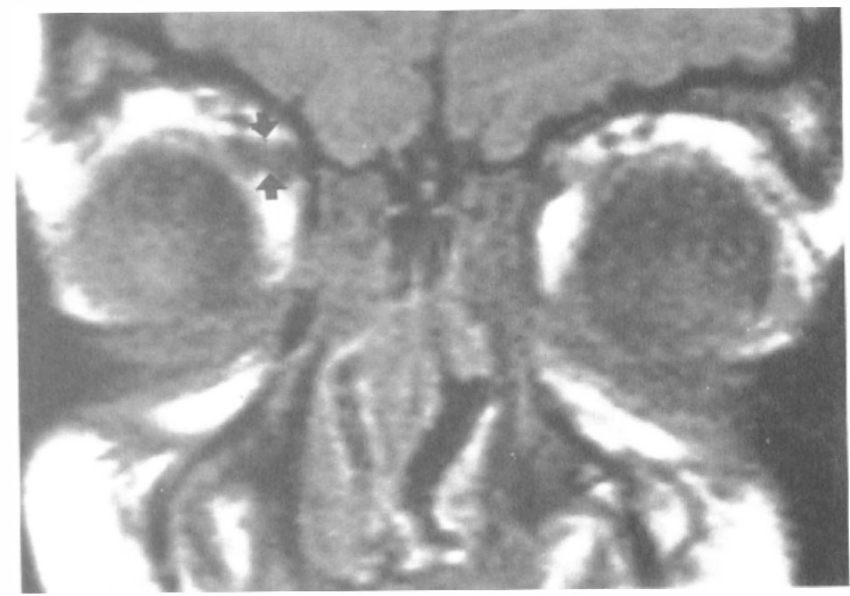

(b)

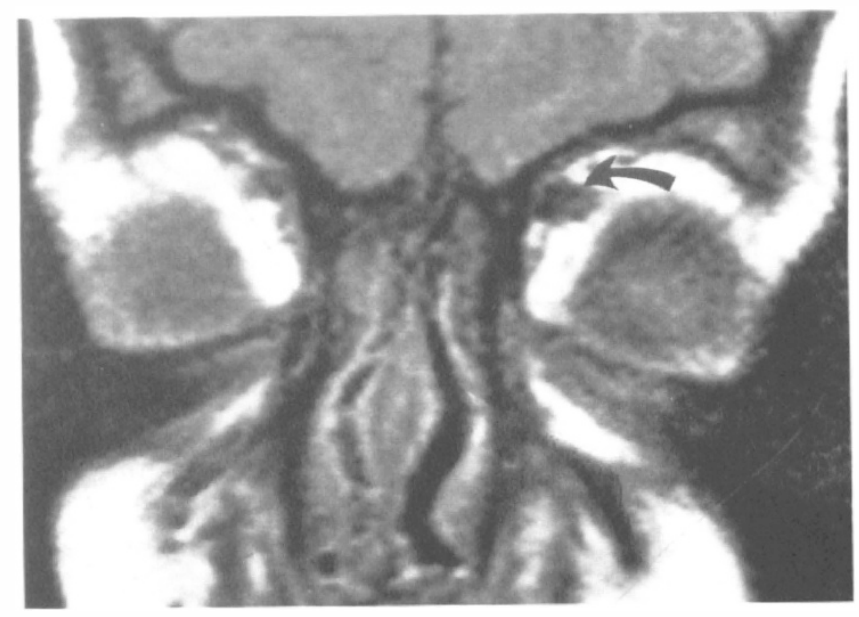

(d)

Fig. 4. Patient 4. Bilateral Brown's syndrome. The axial oblique (a) and coronal images (b) of the right orbit show the irregular tendon-trochlea complex (arrowed). The tendon-trochlea complex (arrowed) of the left orbit is also irregular on axial oblique (c) and coronal (d) images.

eye of patient 4, which developed overcorrection following surgery, tendon-trochlea complex showed enlargement, irregularity and isointensity with the soft tissues observed (Fig. 4a,b). Although his left eye was fully corrected post-operatively, the MR findings of the left tendon-trochlea complex were quite similar to those of the right side (Fig. $4 \mathrm{c}, \mathrm{d}$ ). In the other overcorrected case (patient 8) the tendontrochlea complex was enlarged in width and irregular but showed low signal intensity. In the remaining two operated cases the tendon-trochlea complex was enlarged, irregular and isointense with the soft tissue.

No pathological appearance of the SO muscle bulk was observed in either size or signal intensity.

\section{DISCUSSION}

Greater knowledge of the anatomy of SO muscle and the surrounding tissues has led to the understanding that a 'short anterior sheath' does not have any role in Brown's syndrome, and further that there is no such tissue that could be called an anterior sheath.
Parks ${ }^{8}$ stated that there is a sleeve at the area where the SO tendon penetrates Tenon's capsule and the intermuscular septum envelops the tendon between this sleeve and the insertion.

It was proposed that a tight inelastic tendon may be the cause of Brown's syndrome, and the success of SO tenotomy supported this theory. ${ }^{8,13}$ However, it is a well-documented phenomenon that there may be spontaneous regression in the disease and it does not seem logical that the tightness of the tendon resolves with time in these cases. Plager ${ }^{14}$ regarded congenital SO palsy and Brown's syndrome as a clinical spectrum - the former with an abnormally long tendon and the latter with a short tendon. Spontaneous recovery cannot be explained by this suggestion, since elongation of a congenitally short tendon is unlikely to occur.

In an embryological study Sevel ${ }^{15}$ demonstrated thick trabeculae extending from the tendon to the trochlea that nearly disappear before birth, leaving only fine trabecular remnants. He postulated that the 
persistence of the embryological thick trabeculae may be responsible for Brown's syndrome.

In their detailed anatomical study, Helveston et $a l .{ }^{16}$ described the trochlea as consisting of a cartilage saddle, a dense fibrous condensation that secures the trochlear saddle, an intratrochlear portion of SO tendon and a fibrillo-vascular sheath. Between the tendon's vascular sheath and the trochlear saddle there is a bursa-like structure. It was shown that the central fibres of SO tendon make a $16 \mathrm{~mm}$ vertical excursion. ${ }^{16}$ If this excursion is limited for any reason at the bursa-like structure or by vascular distension in the sheath, this may cause Brown's syndrome.

It has not been possible to differentiate the anatomical structures of the trochlea with MR imaging. We therefore evaluated the tendontrochlea complex in this study. The computed tomography (CT) findings of Brown's syndrome in 3 acquired and in 1 congenital case have been reported previously. ${ }^{17}$ In that study a slight thickening of the reflected tendon was observed in the congenital case and in the acquired cases the thickening was much more profound. In a case with trochleitis, CT showed a soft tissue density at the region of the involved trochlea, a finding similar to ours. ${ }^{18}$ There is only one case report on the MR findings of acquired Brown's syndrome; a 'nodular SO tendon sheath' of low signal intensity was found but the explanation of these findings was not discussed. ${ }^{19}$

Our series is the first to investigate the tendontrochlea complex by means of measurements. It is clear that thickening of the tendon-trochlea complex occurs both in the coronal and, more profoundly, in the axial plane. These findings support the theories that the aetiology of Brown's syndrome lies within the tendon-trochlea complex. Only the anatomical shortness or tautness of the SO tendon is unlikely to cause such an appearance on MR imaging.

Normal tendons appear hypointense on MR examination. However, in 11 of 16 affected eyes the tendon-trochlea complex was isointense to soft tissue. This appearance may occur due to anomalies of any structure in the complex, such as fluid accumulation at the bursa-like structure, thick trabecular remnants or abnormalities in the tendon itself. Another significant finding was the irregular appearance of the tendon-trochlea complex in 14 of 16 involved eyes, which also suggests an abnormality in the tendon-trochlea complex.

We attempted to find a relation between the MR appearance of the tendon-trochlea complex and the response to surgery. Patients who had had previous surgery did not undergo MR examination prior to surgery, but since all of them had had SO tenotomy in sub-Tenon's space we thought that the appearance of the tendon-trochlea complex would not be affected. Although there were few cases with previous surgery, our results indicate that it is not possible to make any comment about the surgical outcome of the patient on the basis of the MR imaging findings. This is also the case for spontaneous improvement.

In conclusion, the MR findings in congenital Brown's syndrome suggested that the primary pathology is located at the tendon-trochlea complex. Clinically the MR examination does not give any predictive clue to the ophthalmologist about the outcome of the disease.

Key words: Congenital Brown's syndrome, Magnetic resonance imaging.

\section{REFERENCES}

1. Girard LJ. Pseudoparalysis of the inferior oblique muscle. South Med J 1956;43:342-9.

2. Parks MM, Brown M. Superior oblique tendon sheath syndrome of Brown. Am J Ophthalmol 1975;79:82-6.

3. Wright KW, Silverstein D, Marrone AC, Smith RE. Acquired inflammatory superior oblique tendon sheath syndrome: a clinicopathologic study. Arch Ophthalmol 1982;100:1752-4.

4. Killian PJ, McClain B, Lawless OJ. Brown's syndrome: an unusual manifestation of rheumatoid arthritis. Arthritis Rheum 1977;20:1080-4.

5. Sanford-Smith JH. Intermittent superior oblique tendon sheath syndrome. Br J Ophthalmol 1969;53:412-7.

6. Sims J. Acquired apparent superior oblique tendon sheath syndrome. Br Orthopt J 1971;28:112-5.

7. Wilson ME, Eustis HS, Parks MM. Brown's syndrome. Surv Ophthalmol 1989;34:153-72.

8. Parks MM. Doyne Lecture. The superior oblique tendon. Trans Ophthalmol Soc UK 1977;97:288-304.

9. Helveston EM. Atlas of strabismus surgery. St Louis: CV Mosby, 1985.

10. Waddell E. Brown's syndrome revisited. Br Orthopt J 1982;39:17-21.

11. Kahan TJ, Smith K, Orton RB, Noel LP, Clarke W, Cadera W. Natural history of presumed congenital Brown syndrome. Arch Ophthalmol 1993;111:943-6.

12. Gregersen E, Rindziunski E. Brown's syndrome: a longitudinal long-term study of spontaneous course. Acta Ophthalmol (Copenh) 1993;71:371-6.

13. Crawford JS. Surgical treatment of true Brown's syndrome. Am J Ophthalmol 1976;81:289-95.

14. Plager DA. Tendon laxity in superior oblique palsy. Ophthalmology 1992;99:1032-8.

15. Sevel D. Brown's syndrome: a possible aetiology explained embryologically. J Pediatr Ophthalmol Strabismus 1981;18:26-31.

16. Helveston EM, Merriam WW, Ellis FD, et al. The trochlea: a study of the anatomy and physiology. Ophthalmology 1982;89:124-33.

17. Mafee MF, Folk ER, Langer BG, et al. Computed tomography in the evaluation of Brown syndrome of the superior oblique tendon sheath. Radiology 1985; 154:691-5.

18. Tychsen L, Tse DT, Ossoinig K, et al. Trochleitis with superior oblique myositis. Ophthalmology 1984;91: 1074-9.

19. Tien RD, Duberg A, Chu PK, et al. Superior oblique tendon sheath syndrome (Brown syndrome): MR findings. AJNR 1990;11:1210. 